\title{
Adherence To Long-Term Complex Medication Regimen After Hospital Discharge From An Academic Geriatric Center: General Practitioners' Attitude
}

\author{
Takeda-Raguin $\mathrm{C}^{1}$, Vogel $\mathrm{T}^{1}$ and Lang $\mathrm{PO}^{* 2,3}$ \\ ${ }^{1}$ Geriatric Rehabilitation Division, Department of Geriatrics, University Hospitals of Strasbourg, France \\ ${ }^{* 2}$ Geriatric and Geriatric Rehabilitation Division, Department of Medicine, University Hospital of Lausanne, Switzerland \\ ${ }^{3}$ Health and Wellbeing Academy, Anglia-Ruskin University, Cambridge, United Kingdom \\ ${ }^{*}$ Correspondence to: Lang PO, Geriatric and Geriatric Rehabilitation Division, Department of Medicine, University Hospital of Lausanne, Switzerland. \\ E-mail: pierre-olivier.lang@chuv.ch
}

Received: August 10, 2016; Accepted: September 17,2016; Published: September 28,2016;

\begin{abstract}
Background: Pharmacotherapy in old age is a very complex process during which adherence to medication recommendations is a key point. Little is known about general practitioner (GP)-related barriers to prescribing medication according to geriatrician recommendations.
\end{abstract}

Objective: To provide a snapshot of the reality of continuity of care and information about factors that modulate GP adherence.

Design: Observational and prospective pilot study.

Setting: One academic center of geriatric rehabilitation and the associated community.

Measurements: Number of prescribed medications at admission time, discharge and four months later. GP attitudes were investigated using a short and specific questionnaire (14 items, 5 minutes to respond).

Participants: Consecutive patients discharged from the Strasbourg hospital geriatric division whose reference GP was known. 150 patients $(84.8 \pm 6.5$ years-old; $69.3 \%$ women) were included and their GPs contacted by telephone. Of the 72 GPs who accepted to participate, 39 GPs answered the first part of the survey and 24 completed the entire survey.

Results: The cohort of 150 patients suffered from $4.9 \pm 2.1$ co-morbidities and took $8.4 \pm 3.2$ medications at admission time and 7.9 \pm 3.0 upon their discharge $(\mathrm{p}=0.038)$. The 39 patients associated with GPs who responded were not different compared to the initial cohort. During their hospital stay, 79 therapeutic modifications were done with a $10.5 \%$ overall reduction in the number of treatments $(\mathrm{p}=0.03)$. Four months later, a further $16.0 \%$ reduction was observed $(\mathrm{p}=0.02)$. The most modified therapeutic classes were ACE inhibitors, ( $\beta$-blockers, statins, oral anticoagulants and proton pomp inhibitors. $(4 \mathrm{months}$ later, patients were back to the initial number of medication even more?).

According to GPs' answers, 58.0\% were satisfied with the medical reports (sent 3 to 4 weeks after hospital discharge) in terms of expectations and the quality. For $91.6 \%$, GPs considered that the therapeutic changes were mentioned in the report, and $83.3 \%$ were properly explained. The usefulness of the report was highlighted by $83.0 \%$ while $46.0 \%$ judged that the optimization of prescribing medications at discharge did not facilitate patient management. At 4 months, $60.9 \%$ of GPs had made further therapeutic modifications. Weakness in transition and continuity of care were particularly underlined by the last open-ended question of the questionnaire.

Conclusion: This pilot study emphasizes that hospital discharge is a crucial time for non-adherence and reinforces the need not only to enhance the quality of transition but also the continuity of care between hospital and community.

Key words: Geriatric patients, anti-hypertensive drugs, anti-platelets, Evidence-based-medicine

\section{Introduction}

The proportion of older people aged 65 years and over is rising faster than any other sector of the general population. This pattern also means there is a steadily increasing number of people with multimorbidity, which generates polypharmacy. Polypharmacy, in turn, is the most consistent predictor of inappropriate prescribing medication, adverse drug events (ADEs) and other drug-related problems, [1] which is now a serious and escalating public health problem.[2]
Pharmacotherapy in old age is a complex process during which errors can occur at any stage. [3, 4] Medication adherence is one of the crucial points of this process and non-adherence increases the risk of negative health outcomes (i.e. poor disease control, therapeutic failure, worsening of functional abilities), more frequent health service utilization and greater health care expenditure, and a higher risk of death.[5-7] 
While older people appear to have a number of risk factors that predispose them to medication non-adherence,[8-9] the majority of published data indicates that age itself is a poor independent predictor. [10] The reasons for medication non-adherence maybe varied and include treatment, patient, health system and prescriber-related factors. $[3,11,12]$ With the aim of optimizing pharmacotherapy in older adults, the understanding of general practitioner (GP)related barriers to prescribing medication according to geriatrician recommendations is, in our opinion, a very important question.

The purpose of this prospective pilot study of GPs was to: (i) provide a snapshot of the reality of continuity of care; (ii) evaluate the participation rate of GPs in such a study; and (iii) provide information about factors that modulate GP adherence with discharge instructions concerning pharmacotherapy. The overall aim was to identify key factors allowing the design of a multicenter intervention study to favor continuity of care between hospital settings and the community.

\section{Materials and methods}

\section{Study design}

This pilot study was divided into two steps. The first step was the retrospective collection of data and analysis of socio-demographic characteristics and changes in medication operated during the stay in hospital, using the final medical report and the patient's medical file. The second step was the prospective collection of medications prescribed 4 months later by GPs and the analysis of the adherence with therapeutic recommendations and changes in medication prescribed at discharge. For this step, the GPs or their secretaries were first contacted by telephone in order to present the study and to organize an appointment to complete the questionnaire.

In case of 3 unsuccessful attempts, the questionnaire was then directly sent by post and/or electronic mail with a letter describing the study (i.e. rationale and objectives). Four months after discharge, the complete list of patient's medication was collected from GPs who replied. The second step was completed either by phone, e-mail or post. For patients who lived in nursing homes, the complete list of medication was obtained directly from the nurse in charge of the patient in the institutional setting. The questionnaire was distributed over a 5-month period from 1st May to 30th September 2014. A reminder by e-mail was sent to all GPs one month after the first contact; there was no reminder by post.

\section{Population study and sampling method}

Data were prospectively collected from 200 consecutive patients aged 75 years or older discharged from the academic geriatric rehabilitation division of the University Hospital of Strasbourg (France) within a 4-month period (1st January to 31st April 2014). Patients were randomly selected throughout the hospitalisation list. Among them, we selected GPs who were taking care of only one patient in order to focus our attention on the factors influencing GPs' prescriptions (patients whom GP were already candidates through another patient were not included). In addition, non-inclusion criteria for all patients were missing data for the medication list either at admission time, at discharge, or after 4 months, and patients for which the GP was not reachable. Criteria for secondary exclusion were withdrawal because of patient's death before the end-point, patients for whom the GP was no longer in charge, patients who were hospitalized at the time of the interview with the GP, and GPs who withdrew from the study or who did not answer to the questionnaire despite three calls and one reminder. As depicted on the flow chart (figure 1), 150 GP/patient duos were identified; 95 were contacted by telephone ( 55 couldn't be contacted); and 72 GPs consented to participate.

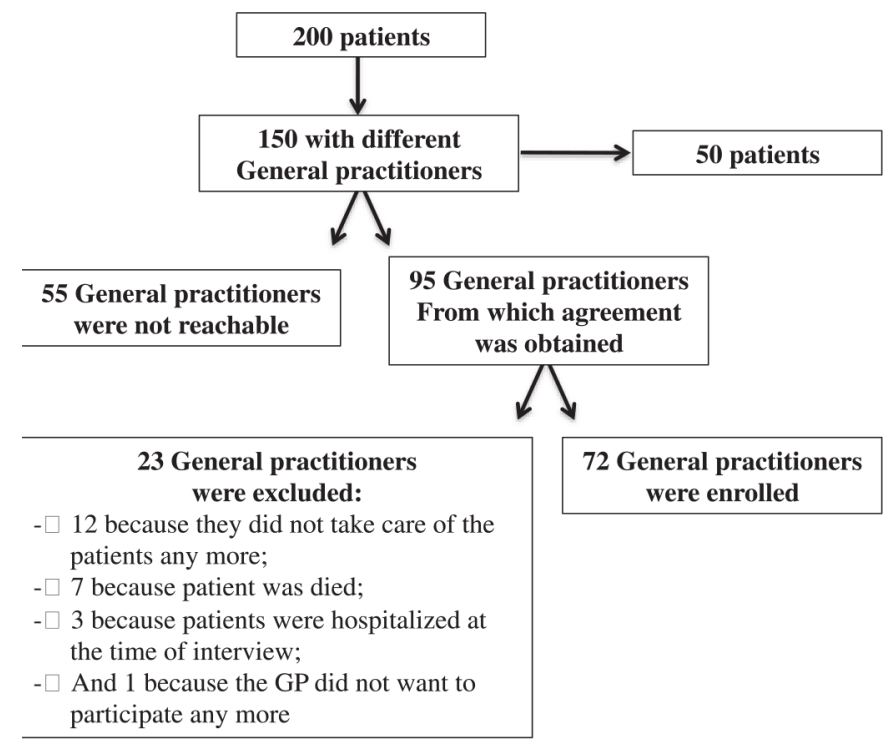

Figure 1: Flow chart of the population study.

\section{Appropriateness of prescribing medication during the hospital stay}

In the present pilot study, all patients enrolled were admitted for rehabilitation following hospitalization either for acute medical conditions or orthopaedic surgery where interdisciplinary healthcare management was provided from admission to discharge. The interdisciplinary team consisted of the geriatric healthcare team with one full-time professor, one assistant senior physician and two fellows for the medical staff, supported by geriatric nurses, ancillary staff, physical therapists and psychologists. Specifically for psychiatric care, when necessary, an additional part-time senior geriatric-psychiatrist complemented the team. This interdisciplinary team designed, implemented and monitored comprehensive care and discharge plans for patients across a care continuum. This approach included a therapeutic plan with the aims of (i) limiting harmful effects through drug-drug or drug-disease interactions; (ii) ensuring the prescription of medications at the right doses and for the correct durations; (iii) systematically balancing the clinical benefit and the risk of adverse drug events (ADE) associated with any prescription with the patient's needs, quality of life and expectations; and (iv) reducing the rate of omission of indicated medications with proven efficacy according to the patient's level of functionality and life expectancy.[4] The medical team was present in the unit on a daily basis, participated in daily medical rounds and weekly interdisciplinary meetings and had direct contact with patients, care givers and patients' families. For every patient admitted, a complete medication history was performed 
with the help of the patient's GP if necessary. At their discharge, specific therapeutic recommendations were transmitted to their GP by telephone ( 1 day around the discharge day according to GP's availability) and via the medical report usually sent by post within 3-4 weeks after discharge.

\section{Elaboration of the questionnaire for General Practitioners (GPs)}

The questionnaire was structured in 5 sections with 13 closedended questions (yes or no) and 1 open-ended question for personal comments. The average time to complete the survey was estimated to be 5 minutes.

The first section was dedicated to the list of medications prescribed 4 months after the hospital discharge. The GP or the referent nurse (if the patient was living in an institutional setting) filled it out. Changes in therapeutics were defined by the modification of at least one pharmaceutical molecule between the list at discharge and 4 months later. The remaining 4 sections were dedicated to GPs. Two sections focused on the prescriber's characteristics (i.e. age, gender, year of installation), his continuing medical training and the type of activity (i.e. own practice, health clinic, and/or practitioner who practices in an institutional setting, training). The last two sections were dedicated to GPs' expectations and more particularly the quality of therapeutic information transmitted ( 4 items); the issue of the complexity of therapeutic recommendations was also addressed ( 2 items).

Before starting the present study, the questionnaire was first tested on $3 \mathrm{GPs}$ (not included in the present study) during the last quarter of 2013 in order to confirm that the questionnaire fitted both the study objectives and was acceptable according to GPs' activity.

\section{Complementary data collection}

At the inclusion time, in addition to the treatment list upon admission, at discharge (M0) and 4 months later (M4), sociodemographic data (age, gender, living conditions) and health status were recorded by either the senior or attending physician. Thus, for each patient, the number co-morbidities was also recorded. For medications, the active pharmaceutical ingredient was considered as a statistical unit (for example, if in one pill two antihypertensive drugs were combined, two active pharmaceutical ingredients were recorded).

\section{Statistical analysis}

Results pertaining to numerical variables are presented as mean \pm standard deviation (SD). For prescriptions of medicines, the median, maximum and minimum numbers of medications are also presented. For categorical variables, number and percentage are presented. Comparative analyses were computed with SAS software (version 9.1, SAS Institute, Cary, NC). Categorical outcomes were tested using the Chi2 (X2) test or Fisher's exact test, and Student's t-test or the Wilcoxon signed rank test for paired samples were used for numerical outcomes as appropriate. The level of significance was set at $\mathrm{p}=0.05$ for all analyses.

\section{Results}

The sample of 150 patients was aged $84.8 \pm 6.5$ years on average (max-min: 94-74years); $69.3 \%$ were women. They suffered, on average, from $4.9 \pm 2.1$ co-morbidities (max-min: 10-1) and took 8.4 \pm 3.2 medications every day at admission time and $7.9 \pm 3.0$ at upon their discharge $(\mathrm{p}=0.038)$ corresponding to 1257 and 1196 active pharmaceutical ingredients respectively. The details of medications prescribed at both these times are presented in figure 2 .

On the 72 GPs initially enrolled in the study, only $78.0 \%$ participated in the first part of the study concerning the medication list 4 months after discharge and $61.5 \%$ answered the sections about their attitudes toward therapeutic recommendations formulated in the final medical report. The corresponding 39 aged patients were not significantly different from the initial sample of 150 in terms of age, gender, co-morbidities and average number of medications at admission and upon discharge ( $\mathrm{p}>0.05)$. These 39 patients accounted at admission time and discharge for $342(8.8 \pm 3.7)$ and $306(7.8 \pm 3.5)$ different medications respectively. Dining the hospital stay in the rehabilitation centre, 79 therapeutic modifications were done by the medical team with a $10.5 \%$ overall reduction of the number of treatment $(\mathrm{p}=0.03)$. Four months after discharge, a further $16.0 \%$

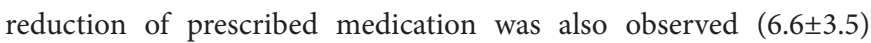
corresponding to 257 molecules for a total reduction of $24.8 \%$ since admission time $(\mathrm{p}=0.02)$. For 17 patients, treatment was strictly similar (43.6\%). As shown in figure 3, the most modified therapeutic class was antihypertensive drugs, which accounted for $33.0 \%$ of therapeutic modifications occurring during the hospital stay. With an overall reduction of $5.8 \%, 77.0 \%$ of antihypertensive treatments adapted during the hospital stay were maintained 4 months later. However, underuse of ACE inhibitors and ( $\beta$-blockers was observed during the stay. Conversely, the number of prescribed anti-calcics and AT2-inhibitors was dramatically reduced. One quarter of statins were stopped during the stay, and this recommendation was maintained 4 month latter in $97.4 \%$ of cases. Oral anticoagulants were dramatically reduced not only during the stay but also after discharge (by $25.6 \%$ in total). With respect to proton pomp inhibitors, the overall reduction during the stay was $18.2 \%$. However, 4 months later, for proton pomp inhibitors, patients were back to initial values or even more $(+4.5 \%)$. The main indication was the combination with anti-platelets.

Among the $39 \mathrm{GPs}$ who answered the questionnaire, $87.5 \%$ were men and $2 / 3$ were aged 50 years or over. On average $15.0 \%$ of their ambulatory patients were aged 70 or over and $91.0 \%$ of them were working at least part time, in institutional settings (e.g. nursing homes, long term care facilities).

With respect to expectations for and the required level of medical report, GPs were (58.0\%) satisfied with a report sent 3 to 4 weeks after hospital discharge. For $91.6 \%$ of them, GPs considered that the therapeutic changes were mentioned in the report and for $83.3 \%$, had been properly explained. The usefulness of the report for the medical follow-up by GPs was highlighted by $83.0 \%$ of the GPs while $46.0 \%$ judged that the optimization of medications prescribed at discharge did not facilitate patient management. At 4 months, $60.9 \%$ of GPs had operated further modifications on the medication list prescribed at discharge; the reasons for are presented in figure 4. 

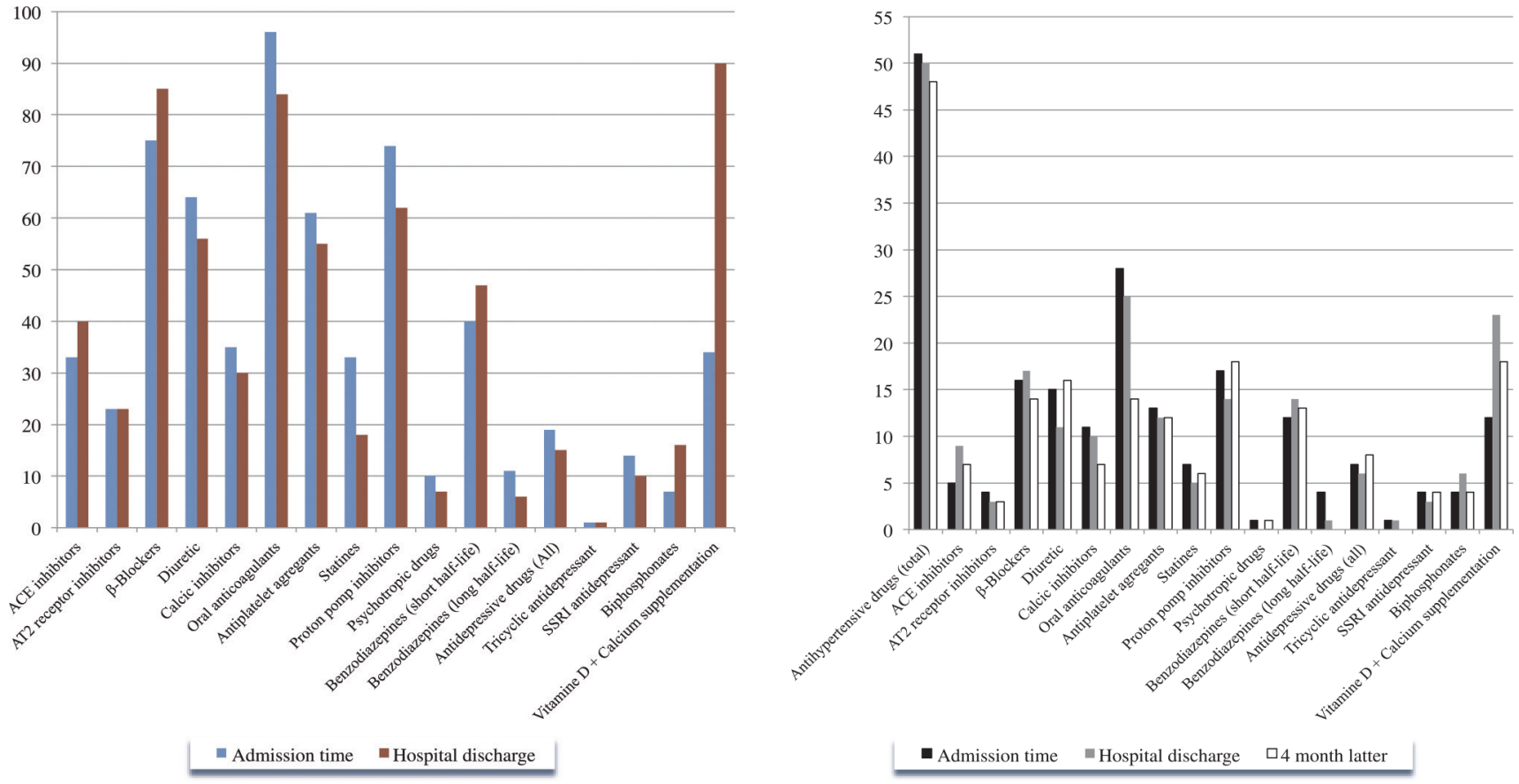

Figure 2 : Descriptive analysis of the number of medications prescribed at admission time and upon discharge among 150 geriatric patients. Results are given according to therapeutic class (ACE, angiotensin-converting enzyme inhibitor; AT2, Angiotensin-2 receptor inhibitor; SSR, specific serotonin-reuptake inhibitor).

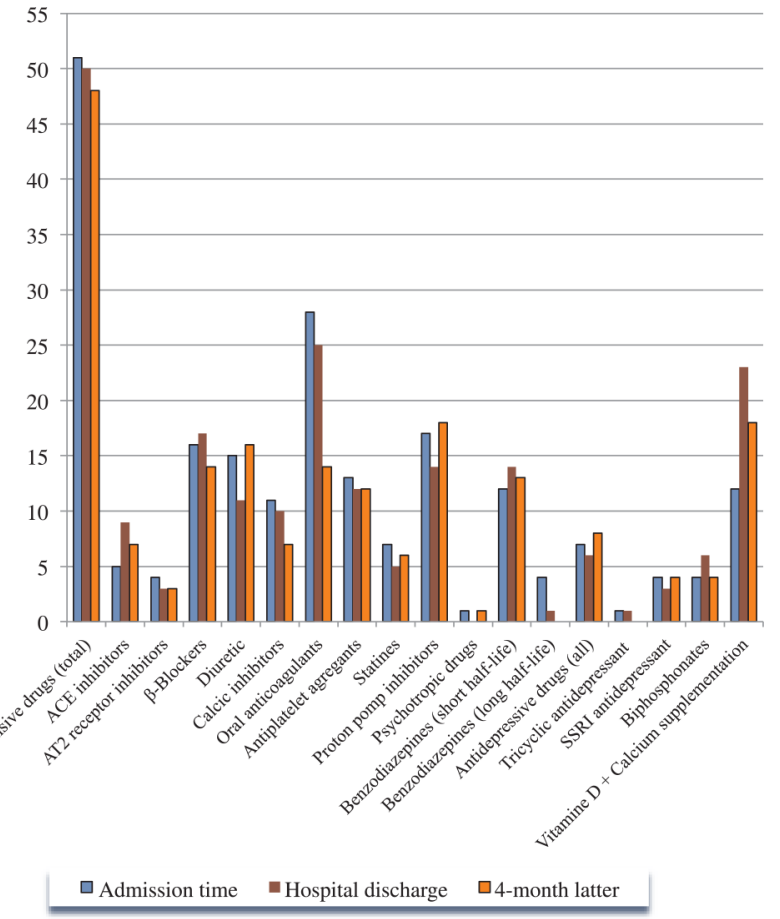

Figure 3 : Descriptive analysis of the number of medications prescribed at admission time, discharge and 4 months lateramong 39 geriatric patients whose GPs responded to the questionnaire. Results are given according to therapeutic class (ACE, angiotensin -converting enzyme inhibitor; AT2, Angiotensin-2 receptor inhibitor; SSRI, specific serotonin-reuptake inhibitor).

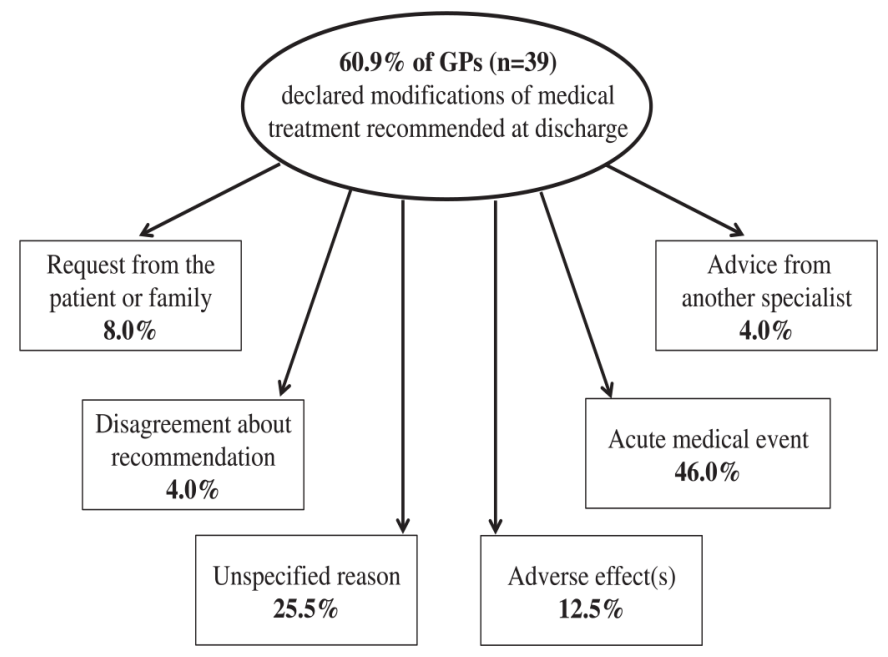

Figure 4: Description of reasons motivating changes in medications prescribed by general practitioners during 4 months following discharge horn the hospital.

The analysis of answers to the open-ended question revealed that most of GPs considered the hospital as expert for optimizing pharmacotherapy. They also underlined that some of the modifications operated were not always appropriate for ambulatory patients. One of the most frequent GP requests was that the hospital physicians keep the same active pharmaceutical ingredient when a treatment was unchanged during the stay. The second one was that a direct contact should be established between the hospital medical team and GPs before any treatment modifications or, at least, when the patient was discharged. 


\section{Discussion}

This pilot study investigated GPs' therapeutic adherence for patients discharged from a geriatric rehabilitation center. The first lesson provided is that, from an initial selected sample of 150 patients, the participation rate by GPs is very low (26.0\%). This result was obtained despite an initial contact by telephone, with three successive attempts and one reminder by e-mail or post with an explicative letter. Moreover, the questionnaire sent was presented in a very short format and was composed by close-ended questions. The second observation is that the therapeutic optimization made during the hospital stay was maintained in only $43.6 \%$ patients. During the stay, the mean number of medications was reduced by $25.0 \%$. Four months after discharge, it was reduced by $15.3 \%$. While nearly $60 \%$ of secondary modifications after discharge were motivated by medication side effects or the occurrence of an acute medical event, $25.5 \%$ were unexplained, $8 \%$ were related to patient or patient's family requests, or on basis of a secondary opinion given by another specialist. While most of the GPs considered the hospital setting as an expert environment for pharmacotherapy optimization, we did not use any tool or specific protocol to drive the optimization and assess its quality during the stay. Commonly, Evidence-Based Medicine (EBM) is used to standardize clinical practice and prevent errors. Based on EBM, guidelines are intended to help clinicians to prescribe appropriately. However, EBM does not often reflect age-specific differences, the high level of medical complexity of older patients, the presence of geriatric syndromes, and general geriatric vulnerability.[13] Guideline-driven prescribing does not help in achieving the goal of appropriate drug treatment and most of the time leads to substantial polypharmacy.[14, 15]

Thus, for patients with multiple conditions and polypharmacy, successful interventions included structured medication review, medication regimen simplification, administration aids and medication reminders, but no firm conclusion in favor of any particular intervention could be made. On average each patient considered in the present study suffered from 4.9 co-morbidities and took 8.4 different medications every day at admission time. Interventions to optimize geriatric pharmacotherapy focused most commonly on pharmacological outcomes (drug appropriateness, adverse drug events, adherence).[3] Systematic reviews, which have analyzed the effect of interventions to reduce inappropriate prescribing across healthcare settings[16-18], have shown that geriatric medicine services (involving geriatrician consultation, comprehensive geriatric assessment, multi-disciplinary geriatric team input into care or specialist case conferences)and multi-disciplinary team interventions (mostly pharmacist and physician collaboration or continuing education)reliably reduce inappropriate prescribing.[3]

At their discharge from the geriatric rehabilitation division, the number of prescribed medications for the 150 patients was significantly reduced, corresponding to 1196 active pharmaceutical ingredients compared to 1257 initially (figure 2). However prescribing appropriateness is not only reducing polypharmacy, but it also encompasses the use of medicines where the clinical benefits outweigh the risk of ADEs. It also includes the use of medicines that reduce the likelihood of drug-drug and drug-disease-interaction, the mis- prescribing of medicines (incorrect dose, frequency and duration) and the under-use of clinically indicated medicines. $[16,19]$

With this in mind, the STOPP/START set of criteria can provide additional benefits to interdisciplinary geriatric team when it is necessary to design an intervention protocol. These criteria cover both common and important instances of potentially inappropriate prescribing and potentially serious errors of prescribing omission in older people.[20] Moreover, since the first iteration in 2008, it has been demonstrated that STOPP criteria medications are significantly associated with ADEs, and STOPP and START criteria applied as an intervention within 72 hours of admission significantly reduce adverse drug reactions and improve medication appropriateness.[19, 21, 22] Moreover, this effect was maintained 6 months post-intervention when subsequent recommendations to the attending clinicians to modify the prescription medications accordingly were notified into final medical reports. This is explained not only because this set of criteria represents the consensus views of a panel of experts in prescribing for older people but also because the clear wording of each criterion gives the opportunity to illustrate easily and precisely all medication changes operated during the stay. This reinforces comments obtained from the open-ended question of the questionnaire sent to GPs. Indeed, GPs had mentioned the quality of the medical report and how it has to be informative about the changes of the treatment regimen during the stay. This is of particular interest because among prescriber-associated barriers to optimal adherence are multi-professional communication and the transition and continuity of care.[3] A range of strategies has been implemented to increase adherence by targeting modifiable provider- related barriers. Although numerous systematic reviews have been published, no conclusion about the effectiveness of strategies to counteract non-adherence could be drawn.[23, 24] Furthermore, interventions were delivered by a single group of professionals and did not respect the geriatric medicine principles of teamwork and multiprofessionality. However, when studies demonstrated statistically significant improvement in adherence, a significant reduction in mortality, better control of chronic conditions and reduced overall healthcare costs were observed.[3] There were many different classes of drugs for which GPs were non-adherent. Cardiovascular treatment was the most adjusted system during the stay, with introduction of ( $\beta$-blockers, ACE inhibitor and reduction of oral-anticoagulant treatment, cholesterol-lowering drugs and calcic-inhibitors. It was also the system that was the most readapted by GPs with proton pomp inhibitors (figure 3). No information in the present study concerned the appropriateness or not of these changes.

Thus, given that optimizing pharmacotherapy can be extraordinarily challenging and complex for physicians, in order to gain maximal benefit from pharmacotherapy and to achieve the best possible quality of life for the patient, one important step towards successful outcomes for complicated pharmacotherapeutic strategies is to favour the patient's capacity to adhere to a complex treatment regimen. For that, it is necessary for GPs to set treatment priorities. However, this step is probably the most difficult because diseasespecific guidelines do not capture the full clinical complexity of the 
patient's pharmacotherapy. Thus, the GP's judgement, experience, skills and attitudes are critical in determining whether or not the patient will have positive therapeutic outcomes from pharmacotherapy.

Despite limitations (e.g. small sample size, no measurement of appropriateness of prescribing recommendations), this pilot study emphasizes that the hospital discharge is an important time for non-adherence to prescribing medication according to geriatricians' recommendations. It reinforces the need not only to enhance the quality of transition but also continuity of care between hospital and community. Thus, it could be tested in a future research protocol the impact of "faxmed" (i.e. a brief summary of the hospital stay with the complete list of medications sent by fax or e-mail the day before or the day of one patient's discharge) or of systematic successive contacts by phone (i.e. at admission time, 48 to 72 hours before discharge, and at discharge) to discuss of medications with GP, and/or of the role that could play nurse practitioners. In that way, it could also be interesting to develop specific guidelines for writing prescriptions for GPs in which, for example, it should be systematically mentioned, in addition to medicines that have been introduced recently, those that have been stopped and those for which the dosage has been adapted with the reason for. This study also suggests the interest of specialized geriatric medical training to improve GPs' skills and knowledge concerning pharmacotherapy in old age.

\section{Acknowledgement}

We would like to sincerely thank all the General Practitioners who made my project of medical thesis project a reality.

\section{References}

1. O'Mahony D, Cherubini A, Petrovic M (2012) Optimizing pharmacotherapy in older patients: a European perspective. Drugs Aging 29: 423-425. [crossref]

2. Ferner RE, Aronson JK (2010) Preventability of drug-related harms - part I: a systematic review. Drug Saf33: 985-994. [crossref]

3. Topinkova E, Baeyens JP, Michel JP, Lang PO (2012) Evidence-based strategies for the optimization of pharmacotherapy in older people. Drugs Aging 29: 477-494. [crossref]

4. Lang PO, Vogt-Ferrier N, Hasso Y, Le Saint L, Drame M, et al. (2012) Interdisciplinary geriatric and psychiatric care reduces potentially inappropriate prescribing in the hospital: interventional study in 150 acutely ill elderly patients with mental and somatic comorbid conditions. J Am Med Dir Assoc13: 406. [crossref]

5. Fallis BA, Dhalla IA, Klemensberg J, Bell CM (2013) Primary medication nonadherence after discharge from a general internal medicine service. PLoS One 2013.

6. Haynes R, Ackloo E, Sahota N, et al. (2008) Interventions for enhancing medication adherence. Cochrane Database Syst Rev.

7. Conn VS, Hafdahl AR, Cooper PS, et al. (2009) Interventions to improve medication adherence among older adults: metaanalysis of adherence outcomes among randomized controlled trials. Gerontologist 49: 447-62.

8. Hughes CM (2004) Medication non-adherence in the elderly: how big is the problem? Drugs Aging 21: 793-811. [crossref]

9. Cooper C, Carpenter I, Katona C, Schroll M, Wagner C, et al. (2005) The AdHOC Study of older adults' adherence to medication in 11 countries. Am J Geriatr Psychiatry 13: 1067-1076. [crossref]

10. Gellad WF, Grenard JL, Marcum ZA (2011) A systematic review of barriers to medication adherence in the elderly: looking beyond cost and regimen complexity. Am J GeriatrPharmacother9: 11-23. [crossref]

11. Osterberg L, Blaschke T (2005) Adherence to medication. N Engl J Med 353: 487- 497. [crossref]

12. McDonald HP, Garg AX, Haynes RB (2002) Interventions to enhance patient adherence to medication prescriptions: scientific review. JAMA 288: 2868-2879. [crossref]

13. Wehling M (2011) Guideline-driven polypharmacy in elderly, multimorbid patients is basically flawed: there are almost no guidelines for these patients. J Am GeriatrSoc59: 376-7.
14. Van den Akker M, Buntinx F, Metsemakers JF, et al. (1998) Multimorbidity in general practice: prevalence, incidence, and determinants of co-occurring chronic and recurrent diseases. J ClinEpidemiol 51:367-75.

15. Boyd CM, Darer J, Boult C, Fried LP, Boult L, et al. (2005) Clinical practice guidelines and quality of care for older patients with multiple comorbid diseases: implications for pay for performance. JAMA 294: 716-724. [crossref]

16. Spinewine A, Schmader KE, Barber N, Hughes C, Lapane KL, et al. (2007) Appropriate prescribing in elderly people: how well can it be measured and optimised? Lancet 370: 173-184. [crossref]

17. Kaur S, Mitchell G, Vitetta L, et al. (2009) Interventions that can reduce inappropriate prescribing in the elderly: a systematic review. Drugs Aging 26:1013-28.

18. Steinman MA, Hanlon JT (2010) Managing medications in clinically complex elders: "There's got to be a happy medium". JAMA 304: 1592-1601. [crossref]

19. O'Mahony D, Gallagher P, Ryan C, Byrne S, Hamilton H, et al. (2010) STOPP \& START criteria: A new approach to detecting potentially inappropriate prescribing in old age. EurGeriatrMed 1: 45-51.

20. Lam MP, Cheung BM (2012) The use of STOPP/START criteria as a screening tool for assessing the appropriateness of medications in the elderly population. Expert Rev ClinPharmacol5: 187-197. [crossref]

21. Hamilton H, Gallagher P, Ryan C, Byrne S, O'Mahony D, et al. (2011) Potentially inappropriate medications defined by STOPP criteria and the risk of adverse drug events in older hospitalized patients. Arch Intern Med 171:1013-9.

22. Gallagher PF, O'Connor MN, O'Mahony D (2011) Prevention of potentially inappropriate prescribing for elderly patients: a randomized controlled trial using STOPP/START criteria. ClinPharmacolTher89: p. 845-54.

23. Conn VS, Hafdahl AR, Cooper PS, et al, (2009) Interventions to improve medication adherence among older adults: meta- analysis of adherence outcomes among randomized controlled trials. Gerontologist 49: 447-62.

24. Haynes R, Ackloo E, Sahota N, et al. (2008) Interventions for enhancing medication adherence. Cochrane Database SystRev4: CD000011.

\section{Citation:}

Takeda-Raguin C, Vogel T and Lang PO (2016) Adherence To Long-Term Complex Medication Regimen After Hospital Discharge From An Academic Geriatric Center: General Practitioners' Attitude. Internal Med Res Open J Volume 1(1): 1-6 